\title{
Non-Invasive in vivo Screening of Oral Malignancy Using Laser-Induced Fluorescence Based System
}

\author{
DOI: $10.17691 / \mathrm{stm} 2018.10 .1 .02$
}

Received November 7, 2017

\begin{abstract}
A. Patil, PhD, Associate Professor, Center for Biophotonics, Department of Atomic and Molecular Physics';
V.K. Unnikrishnan, PhD, Associate Professor, Center for Biophotonics, Department of Atomic and Molecular Physics';

R. Ongole, MDS, Professor, Department of Oral Medicine and Radiology2;

K.M. Pai, MDS, Professor and Dean, Department of Oral Medicine and Radiology3;

V.B. Kartha, PhD, Professor, Adviser, Center for Biophotonics, Department of Atomic and Molecular Physic';

S. Chidangil, PhD, Professor and Head, Center for Biophotonics, Department of Atomic and Molecular Physics ${ }^{1}$
\end{abstract}

${ }^{1}$ Manipal Academy of Higher Education, Manipal, Karnataka-576104, India;

${ }^{2}$ Manipal College of Dental Sciences, Mangalore, Manipal Academy of Higher Education,

Manipal, Karnataka-576104, India;

${ }^{3}$ Manipal College of Dental Sciences, Manipal, Manipal Academy of Higher Education, Manipal,

Karnataka-576104, India

About 4000 spectra from oral cavity sites have been recorded in vivo from 380 volunteers, under normal $(n=133)$, potentially malignant $(n=155)$, and malignant $(n=92)$ conditions, by excitation with $325 \mathrm{~nm}$ CW He-Cd laser. The analyses of the spectra show that the overall spectra arise from about 7-8 bands from different molecular species. In healthy condition (from subjects with no abnormal oral conditions, including tobacco use), buccal mucosa, lip underside, and tongue bottom give spectra very similar to each other, while tongue top, tongue lateral, and palate give spectra different from these as well as from each other. Under potentially malignant and malignant conditions all sites give spectra which are noticeably different for different sites and under different conditions. It is therefore advisable to use separate reference data sets of normal, potentially malignant, or malignant conditions for different sites for optical diagnostic applications.

Key words: laser-induced fluorescence; screening oral malignancy; oral cancer.

How to cite: Patil A., Unnikrishnan V.K., Ongole R., Pai K.M., Kartha V.B., Chidangil S. Non-invasive in vivo screening of oral malignancy using laser-induced fluorescence based system. Sovremennye tehnologii v medicine 2018; 10(1): 15, https://doi.org/10.17691/ stm2018.10.1.02

\section{Introduction}

Considerable work has been carried out by many groups during the last several years to develop the technique of tissue fluorescence spectroscopy as a suitable method for early diagnosis of oral cancer [1-20]. Almost all these studies were done on buccal mucosa, though other sites in the oral cavity, like tongue, lips, and upper and lower palates, also are often found to be sites of malignancy. It is well known that these different sites have tissue structure differing from one another [21], and as such, their spectra, though superficially similar, exhibit noticeable differences. This is more so in oral diseases which may precede potentially malignant and malignant conditions. Proper interpretation of the observed fluorescence spectra of these different sites is thus necessary for, not only to develop suitable optical methods for diagnosis, but also to understand the complex biological processes that take place during induction, progression, and regression of oral malignancy, and follow up in therapy. In earlier studies on fluorescence spectra of different sites in the oral cavity mostly spectra above $450 \mathrm{~nm}$ have only been recorded, and excitation wavelengths have also been limited to $337 \mathrm{~nm}$ and above $[6,9,16,20]$.

Of the various biochemical species present in tissues, the amino acids such as tryptophan, tyrosine, and phenylalanine, do not absorb (so do not give any fluorescence) excitation above $300 \mathrm{~nm}$. Pyridoxine, lipo-pigments, collagen, elastin, nicotinamide adenine dinucleotide $(\mathrm{NADH})$, porphyrins, and flavins have strong absorption bands in the $300-500 \mathrm{~nm}$ range, giving rise to fluorescence in the $350-700 \mathrm{~nm}$ range [2]. Though all these species have fairly broad absorption bands (40-50 nm half width FWHM), which overlap in several cases, excitation at a given wavelength may not give fluorescence from all species which absorb at that wavelength. For example, excitation around $275 \mathrm{~nm}$, gives mainly protein spectrum, though NADH and flavins also have strong absorption at that wavelength. A change

Corresponding author: Santhosh Chidangil, e-mail: santhosh.cls@manipal.edu 
in excitation wavelength of $20-30 \mathrm{~nm}$ can produce considerable changes in the fluorescence spectrum. The problem is more acute when pulsed lasers are used for excitation, and spectra are recorded after some delay from the laser pulse, since a few nanoseconds jitter in the laser or intensified charge-coupled device (ICCD) trigger and gate, can give spectra which may vary from time to time, due to fast decay of the fluorescence of some of the species [17]. Some workers have used excitation wavelengths around $400 \mathrm{~nm}$ and have shown that fluorescence in the $500-700 \mathrm{~nm}$ region, from porphyrins, is capable of discrimination between normal and malignant conditions [6, 20]. However, this difference presumably from increased angiogenesis in malignancy, may lead to misleading results, since conditions like inflammation of tissue, wound healing, and bacterial invasion produce similar situations. Only flavins will absorb above $450 \mathrm{~nm}$ to give fluorescence in the 500 $600 \mathrm{~nm}$ region. Unfortunately, normal, and malignant conditions show very similar fluorescence in this case, any changes observed being in absolute intensity only, and diagnostic accuracy from such spectra may vary from user to user, unless all instruments are accurately calibrated for absolute intensity measurements. In general, radiation in the wavelength region of 300-350 nm may be more suitable for laser-induced fluorescence (LIF) diagnosis of oral cavity malignancy [17].

Excitation wavelengths in the range $300-450 \mathrm{~nm}$, have been used in the studies by different groups [1520]. Fluorescence spectra from different sites of the oral cavity, in the range $455-867 \mathrm{~nm}$, using excitation in the $350-450 \mathrm{~nm}$ region, have also been studied for normal, healthy subjects [14]. In many of these studies, only a limited number of subjects/samples were used, mostly in vitro, and combined data from all the sites (or selected sites only) were used for evaluating the suitability of fluorescence spectrum for discrimination of

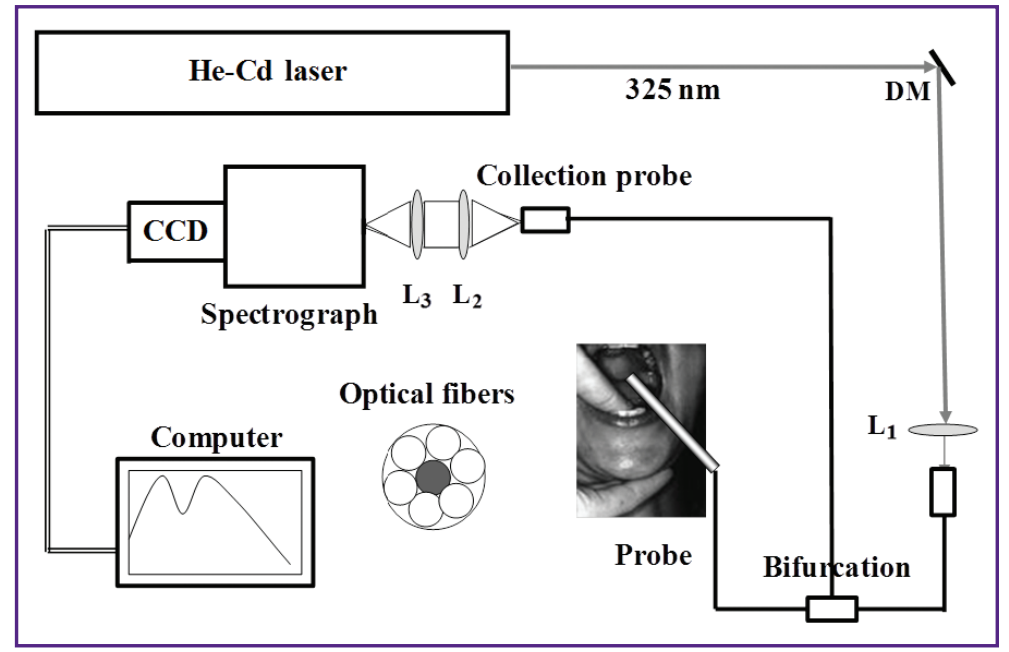

Figure 1. Schematic layout of portable laser-induced fluorescence set up

DM: dichroic mirror; $L_{1}, L_{2}, L_{3}$ : lenses normal, potentially malignant, and malignant conditions. Not much effort was made to understand the basic spectral differences or the desirability or otherwise of using combined data from different sites, for diagnosis. We have studied the fluorescence spectra in vivo from different sites of the oral cavity in normal, potentially malignant, and malignant conditions using a fairly large number of subjects in each case. In our earlier studies we have explored the effect of using different excitation wavelengths 275-425 nm [17], for the present study, excitation by $325 \mathrm{~nm}$ from a He-Cd laser only was used for two reasons; one, the use of a $\mathrm{CW}$ laser needs no ICCD and any effects of time delays between laser and detector in pulsed laser-ICCD trigger combination is avoided and two, excitation around $310-330 \mathrm{~nm}$ causes fluorescence from many components present in tissue compared to excitation at higher wavelengths [2]. The spectra from the different sites were analyzed by curve fitting, difference spectral calculations, and simulation to get a better understanding of changes in tissue spectra as the system proceeds from normal to malignant state through different potentially malignant conditions like leukoplakia, erythroplakia, and sub-mucosal fibrosis. The results of our studies are presented and discussed in this paper.

\section{Materials and Methods}

Laser-induced fluorescence instrumentation. A schematic layout of our portable laser fluorescence set up used in the in vivo studies is shown in Figure 1. The system consists of a CW He-Cd laser ( $5 \mathrm{~mW}$ ) (IK5351R and KR1801C; Kimmon, Japan), an Acton spectrograph (ARC-SP150; Acton, USA) with CCD (DU920N-BU; Andor, Ireland), and a fiber optic probe fabricated in our laboratory. The probe consists of 7 optical fibers (NA=0.22, core diameter $200 \mu \mathrm{m}$ ) (Ocean Optics, USA) with the center fiber used for excitation and the surrounding 6 fibers for fluorescence collection. The fiber tip is enclosed in a removable stainless steel cap with a quartz window (1 $\mathrm{mm}$ thick) which is replaced after use on each subject. The spectrograph was calibrated with a Hg-Ar lamp (HG-1; Ocean Optics, USA). The entire system has been shown to be very stable, the mean variation in wavelength over several months being around $1 \mathrm{~nm}$. The system was thoroughly tested and optimized for in vivo measurements [22].

Samples and spectra acquisition. LIF set up was kept in two locations for the oral cancer screening, i.e. first location was Shirdi Sai Baba Cancer Hospital and Research Center, affiliated to Kasturba Hospital, Manipal, Karnataka, India and the other was Manipal College of Dental Sciences, Manipal and Mangalore, Karnataka, India. We could screen total 380 volunteers (i.e. 133 normal, 155 potentially malignant, and 92 
Table 1

Data distribution among different conditions and anatomical sites

\begin{tabular}{|c|c|c|c|c|c|c|c|c|c|c|c|c|c|c|c|c|}
\hline \multirow{3}{*}{ Class } & \multicolumn{2}{|c|}{ Normal } & \multicolumn{10}{|c|}{ Potentially malignant } & \multicolumn{4}{|c|}{ Malignant } \\
\hline & \multirow{2}{*}{ 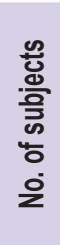 } & \multirow{2}{*}{ 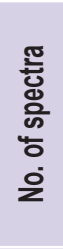 } & \multicolumn{2}{|c|}{ Leukoplakia } & \multicolumn{2}{|c|}{$\begin{array}{c}\text { Speckled } \\
\text { leukoplakia }\end{array}$} & \multicolumn{2}{|c|}{ OSMF } & \multicolumn{2}{|c|}{ Lichen planus } & \multirow{2}{*}{ 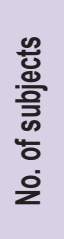 } & \multirow{2}{*}{ 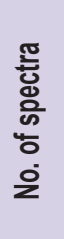 } & \multirow{2}{*}{ 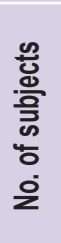 } & \multirow{2}{*}{ 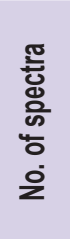 } & \multirow{2}{*}{ 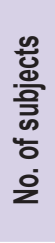 } & \multirow{2}{*}{ 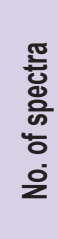 } \\
\hline & & & 은 $\frac{\frac{0}{0}}{\frac{d}{0}}$ & 은 总 & 은 $\frac{\frac{m}{0}}{\frac{.0}{0}}$ & $2 \frac{\text { 윻 }}{\frac{\pi}{4}}$ & 은 $\frac{\frac{0}{0}}{\frac{0}{0}}$ & 은 党 & 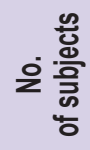 & 은 营 & & & & & & \\
\hline Buccal mucosa & 113 & 453 & 46 & 137 & 13 & 31 & 59 & 227 & 19 & 55 & 91 & 284 & 45 & 115 & 85 & 293 \\
\hline Tongue lateral & 113 & 226 & 3 & 5 & 2 & 2 & 43 & 84 & 5 & 15 & 104 & 203 & 30 & 72 & 81 & 145 \\
\hline Lip & 113 & 180 & 10 & 16 & 3 & 4 & 47 & 49 & 3 & 7 & 92 & 92 & 13 & 25 & 80 & 83 \\
\hline Tongue tip & 113 & 113 & 1 & 1 & 0 & 0 & 35 & 35 & 0 & 0 & 116 & 116 & 9 & 10 & 82 & 83 \\
\hline Tongue top & 113 & 118 & 2 & 4 & 0 & 0 & 35 & 35 & 3 & 4 & 115 & 120 & 10 & 15 & 79 & 79 \\
\hline Tongue bottom & 113 & 113 & 2 & 2 & 0 & 0 & 44 & 45 & 0 & 0 & 107 & 107 & 15 & 27 & 71 & 72 \\
\hline Palate & 64 & 64 & 3 & 5 & 0 & 0 & 1 & 2 & 0 & 0 & 0 & 1 & 7 & 20 & 0 & 0 \\
\hline
\end{tabular}

$\mathrm{N}$ o t e. Total number of subjects - 380 (133 normal, 155 potentially malignant, and 92 malignant). Total number of spectra recorded — 3994 ( $\approx 10$ spectra from each subject). OSMF — oral submucous fibrosis.

malignant cases) who visited these centres. Among 380 volunteers, all normal and except few potentially malignant cases diagnosed clinically by well experienced clinicians of Oral Medicine and Radiology Department of Manipal College of Dental Sciences, Mangalore, India. Potentially malignant and all malignant cases were confirmed by taking post LIF screening biopsy and histopathology report. Ethical clearance was obtained from MU Ethics Committee.

A total of 3994 fluorescence spectra were recorded with LIF system (50 accumulations with integration time of $0.02 \mathrm{~s}$ ) in vivo from 380 volunteers with 3 categories (133 normal, 155 potentially malignant, and 92 malignant). The protocol for taking spectra was as follows: calibrate the spectrograph and take a background spectrum, with the laser on and the probe tip against a dark background to record the background, which is subtracted from all spectra. Wash the mouth with ordinary water 3 times; rinse with saline 2 times; rinse again with HPLC grade distilled water; record at least two in vivo fluorescence spectra each, from the lesion site and corresponding unaffected site. The probe was always placed in contact and as close to normal as possible with reference to the tissue surface, with minimum applied pressure. The sample details are given in Table 1. The spectral data distribution is summarized in Figure 2.

Data processing and data analysis. Details of our data processing and data analysis (GRAMS/AI, Thermo Fisher Scientific, USA) are given elsewhere [23-31]. In our analyses all spectra were subjected to pre-processing which involve background subtraction, smoothing, baseline correction, and normalization. The baseline correction reduces shift in signal level due to changes

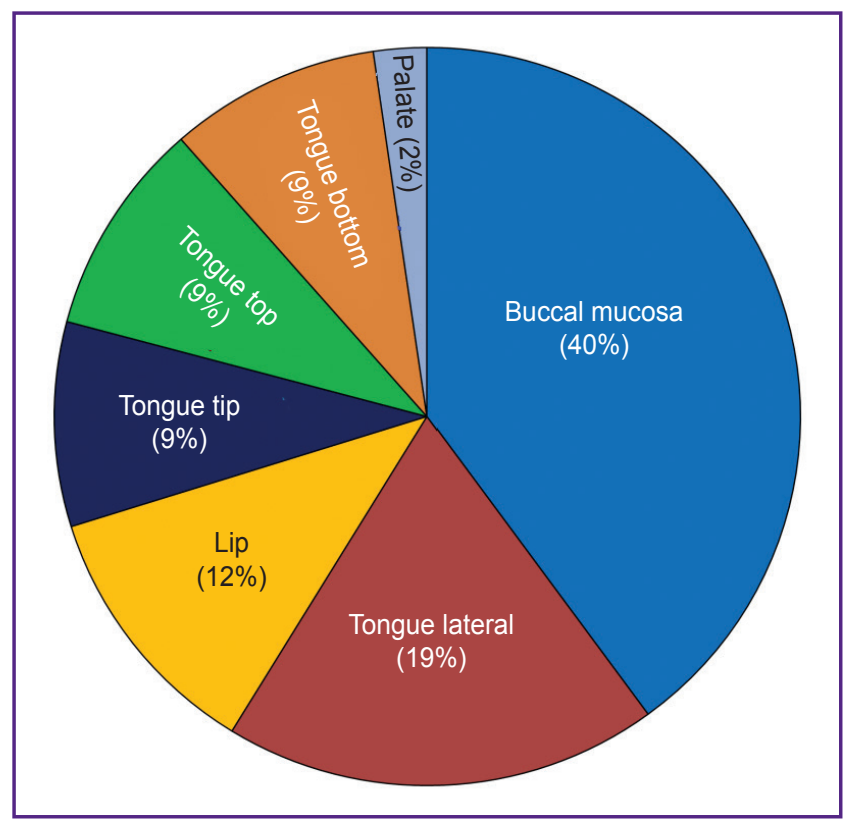

Figure 2. Spectral data distribution from 380 subjects

in efficiency of the system like grating efficiency and CCD sensitivity. Normalization is done with reference to the peak of the strong fluorescence band around $460 \mathrm{~nm}$. Though information on absolute intensities is lost in this normalization, it has two advantages; one, it gives immediate direct information on relative intensity changes; two, it enables to a good extend transfer of data between institutions using different, but comparable, 
instrumentation without need for precise intensity calibration.

Though clinical samples like tissue contain many bio-molecules only a few are present in relatively large amounts. Under normal conditions they will all be present within specific concentration ranges in all samples of a given type, for example, buccal mucosa. Further, at the shorter wavelengths used for fluorescence spectroscopy, the radiation penetrates only a few hundred microns, and the resultant fluorescence, when measured at the illumination point, will be mostly from this top layer [32]. It is thus possible to represent the total profile as a sum of fluorescence profiles of combinations of 2-3 species, each combination varying only slightly from sample to sample. The consequence of this is that if we take the mean of several profiles of a given class, buccal mucosa from normal subjects, each individual member of the class will differ from the mean only by small amounts.

\section{Results and Discussion}

Oral cavity sites - normal conditions. As mentioned earlier, though there are noticeable differences between normal and malignant conditions in absolute intensities of the fluorescence spectra, it may not be convenient to use them for diagnostic applications. Instrumental components (spectrograph, detector, probes) and experimental conditions may vary from user to user and for transferability of absolute intensities between users accurate calibration will be required. On the other hand, relative intensities can be made transferable provided they are normalized to a common specification. We have, therefore normalized all spectra to the peak intensity of the band in the $450-460 \mathrm{~nm}$ region. This band was chosen because, it remained more or less unchanged in shape, and, since intensity variations will remain minimum at the peak, any small errors in wavelength calibration

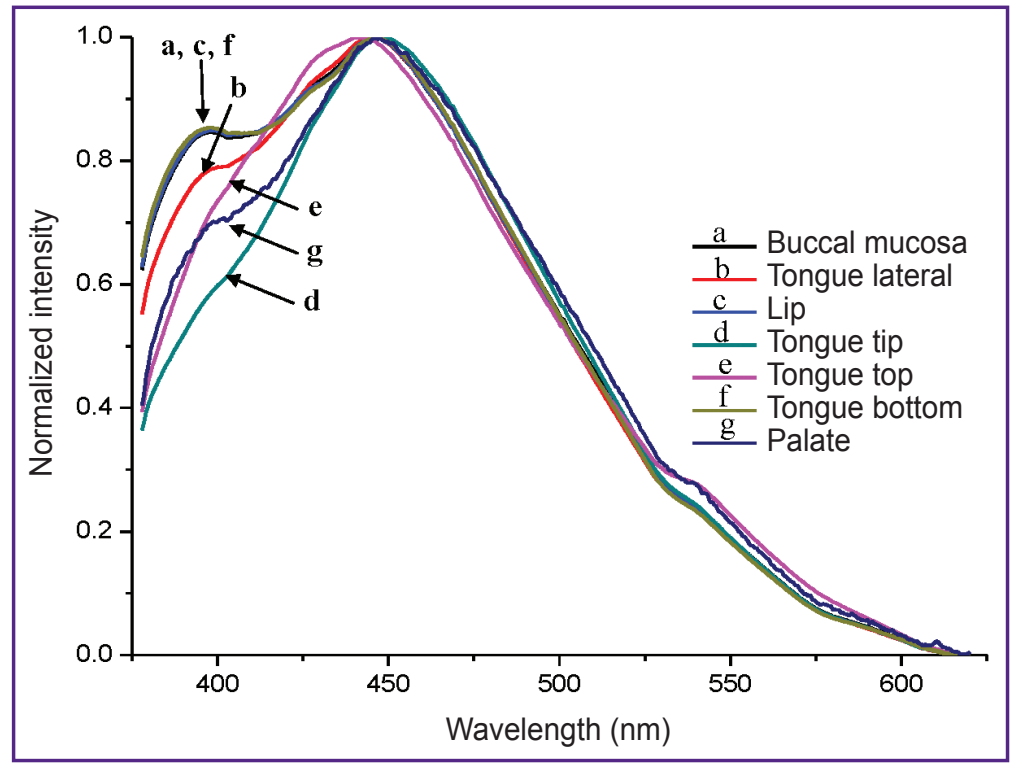

Figure 3. Mean spectra of oral cavity sites - normal will cause only minimum error in normalization. Choice of a fixed wavelength for normalization may result some times in use of points showing rapid intensity variations, which can cause considerable error, since any small difference in wavelength calibration can change intensity considerably from run to run.

The mean spectra of the different sites from clinically normal subjects - no oral diseases, no tobacco habits recorded in vivo are shown in Figure 3. De Veld et al. [14] have measured the auto-fluorescence spectra from 13 sites in the oral cavity for healthy oral mucosa. At $405 \mathrm{~nm}$ excitation, with spectra recorded in the 455$867 \mathrm{~nm}$ range, they have shown that dorsal side of tongue (tongue top), and vermillion border of lip only were noticeably different from 11 other sites which more or less resembled each other quite well. They had concluded that the remaining 11 locations can be combined in a reference data base for future lesion diagnostics. The differences seen by them were mainly of two types; one, appearance of proto-porphyrin type bands for tongue top, and two, differences in intensities for the $490 \mathrm{~nm}$ band when normalized by area of fluorescence spectrum. They have not apparently studied spectra below $450 \mathrm{~nm}$.

An examination of Figure 3 shows that in the normal tissue, at least three quite strong fluorescence peaks are observed by excitation with $325 \mathrm{~nm}$ radiation. Though there are broad similarities in the spectra from different sites, there are also large differences between some of the sites, for example, between tongue tip/tongue top, palate, and the rest. Lower lip and tongue bottom are similar to buccal mucosa, and were considered to be of the same type as buccal mucosa [14]. Excitation with $325 \mathrm{~nm}$ gave bands at 390, 430, and $455 \mathrm{~nm}$, none of which were observed by $405 \mathrm{~nm}$ excitation. The spectra also show possible weak intensity coming from the $500 \mathrm{~nm}$ band [14].

The differences are brought out more clearly in Figure 4 where the difference spectra (mean normal buccal mucosa - mean normal other site) are shown. For normal condition, this indicates more fluorescence compared to buccal mucosa in the collagen region for all sites except lip and tongue bottom. Tongue top and bottom mainly differ by the presence of numerous, slender, filiform papillae with cornified tips which are present only at the top, bottom having a very smooth mucosa. Even for tongue lateral site, which is considered similar to buccal mucosa there are noticeable differences in all regions which are evident from the difference spectra.

The difference spectra clearly show that the spectra of the different sites differ at many places across the entire wavelength region, as indicated by the several small peaks, shoulders, and dips (eg. 378, 390, 400, $430,455,478,500,530$ etc.) seen clearly in the difference spectra (See Figure 4). This 


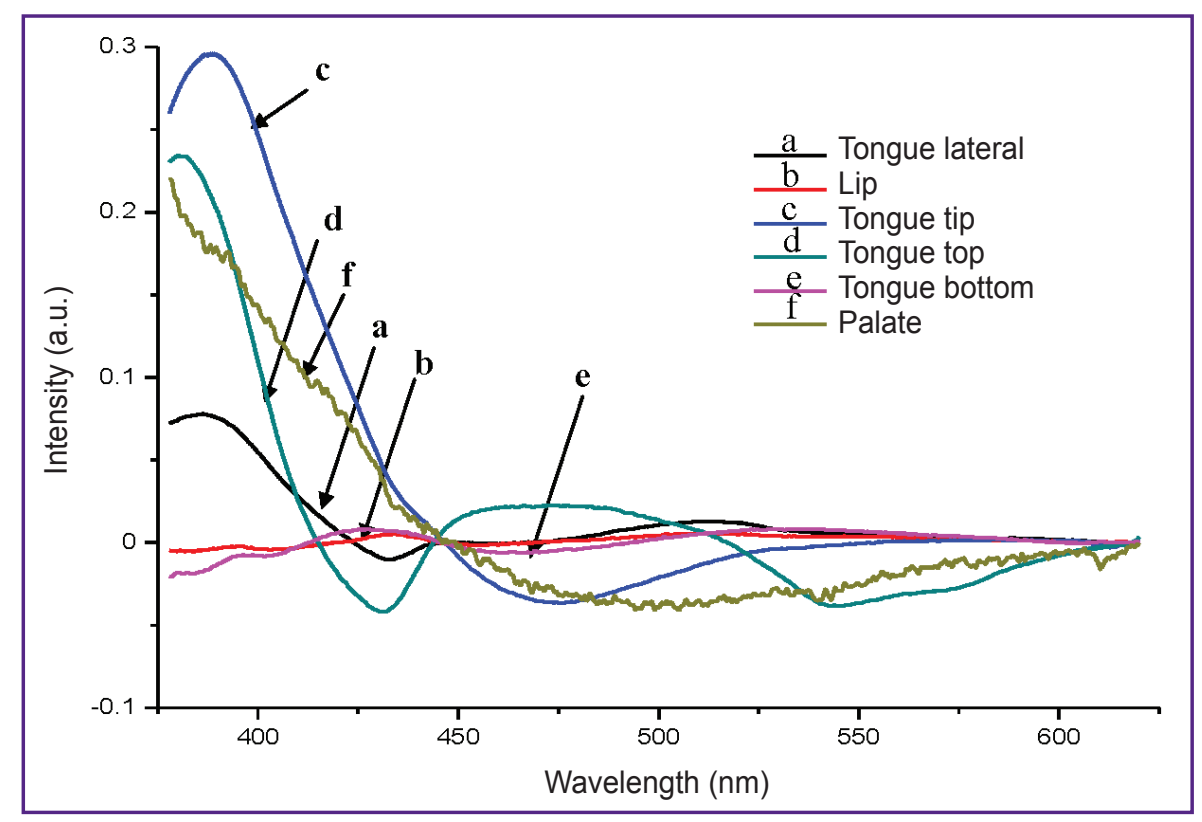

Figure 4. Difference spectra of normal oral cavity sites (mean buccal mucosa mean of other site)

indicates that the overall spectrum cannot be considered as a superposition of just 2 components [11] (collagen and $\mathrm{NADH}$ ), but is a combination of several different closely similar overlapping bands, since, otherwise, the difference spectra would have appeared with only a few symmetric, peaks and dips and the relative intensities in different sections in the spectra would have been maintained in the difference spectra also. But as seen from Figure 4 (buccal mucosa - tongue top), the $450-500 \mathrm{~nm}$ range is negative, while the $530-600 \mathrm{~nm}$ is positive. If this entire range is to be attributed to only $\mathrm{NADH}$ [11], the difference spectrum in the whole range would have been either wholly positive or wholly negative. It should be noted at this point, that, even the dips around 540 and $577 \mathrm{~nm}$ that are supposed to be due to hemoglobin absorption [14], may not be really so, since they are not observed for the others, and have also been observed for cell pellets [11] which are unlikely to have any hemoglobin.

From Figure 4 it is clear that the major difference between other sites and buccal mucosa, lip underside, and tongue bottom, is a substantial decrease in relative fluorescence in the collagen region. Unfortunately, this is the same characteristic which discriminates malignant buccal mucosa from normal. It is thus obvious from even primary considerations that the other sites cannot be clubbed with these three, without incurring possible wrong diagnosis in detection of malignancy. In principal component analysis (PCA) (or any other statistical pattern analysis), parameters like scores of factors, characterizing fluorescence spectra of each class are first derived to discriminate between different classes. Physiological samples come from subjects with widely varying life styles, food habits, social and economic status, age (pre- and post-menopause), substance abuse (tobacco, alcohol), etc. As a result, any diagnostic test will give not single values for the decision-making parameters but values spanning a distribution (eg. fasting glucose for normal subjects 60 to $110 \mathrm{mg} / \mathrm{dl}$ ). This effect will be more in disease, depending upon stage of the disease at the time of the test. Pattern analysis methods like PCA, will thus give a range of values for the parameters determining the spectra, even for a single class of samples, and clubbing together different types will increase the probability of wrong classification.

To evaluate how far one is justified in combining the data of all sites to form a "Reference Database", we did a PCA of the combined data. Using 12 factors to fit the data, it was found that the first 6 factors contributed to $99.7 \%$ of the variance of the samples from the "mean". Figure 5 shows the relative contributions of these 6 factors. It was found that factor 1 contributes $84 \%$, factor 2 another $10 \%$, and factor 3 about $4 \%$, the rest contributing less than $1 \%$ each. It is interesting to note that the number of significant factors also indicate that 3 major and 3 minor independent spectral combinations are required to express the spectrum fairly well. Since, as mentioned earlier, each factor can correspond to a combination of more than one molecular species, it is clear that more than 5-6 molecular components are required to reproduce the spectral emission for the different sites. It can be seen that the major contribution (factor 1) arises out of differences in the collagen region, and to some extent the $\mathrm{NADH}-$ flavin regions as expected from the spectral distribution. If we now look at the distribution of the scores of factor 1 for different anatomical sites of oral cavity shown in Figure 6 suggests that while buccal mucosa, lip 
underside, and tongue bottom may be clubbed together for normal tissues, the palate, tongue tip, and tongue top form classes separate from buccal mucosa. This has important implication for early, objective diagnosis.

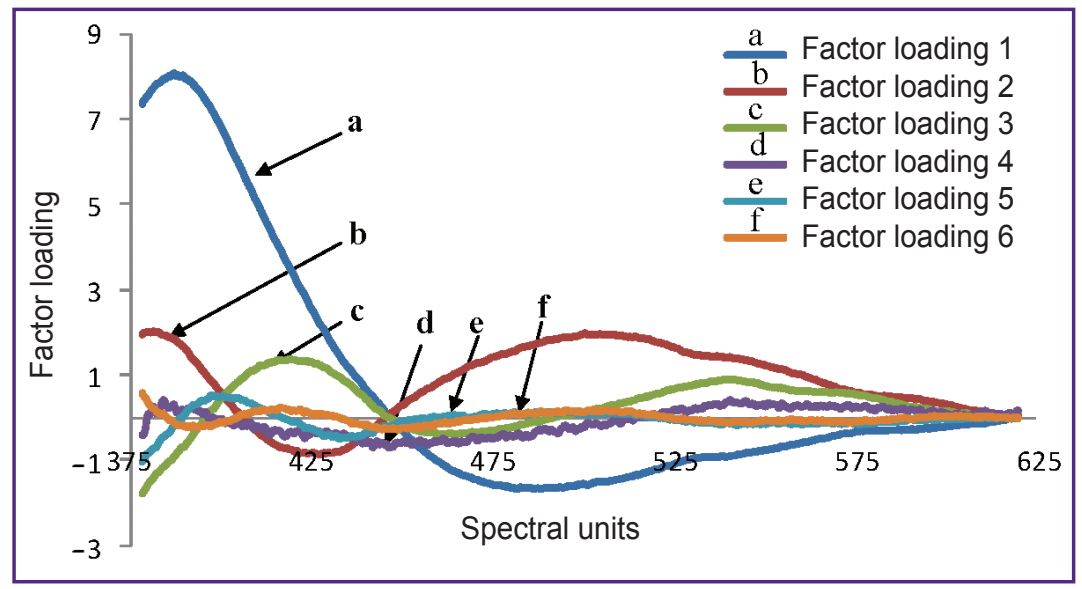

Figure 5. Factor loadings for normal oral cavity sites

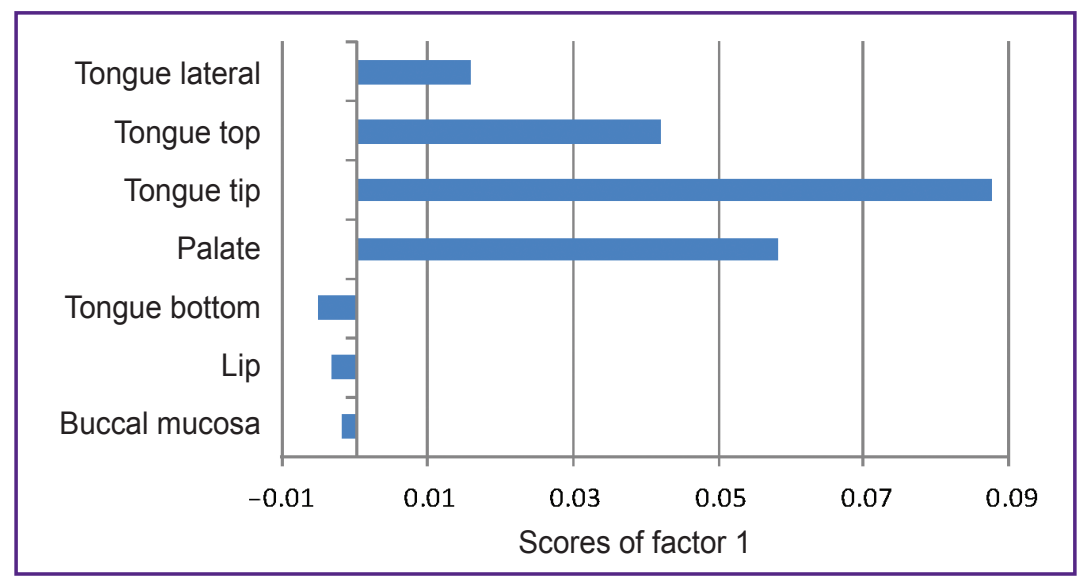

Figure 6. Distribution of scores of factor 1 vs normal oral cavity sites

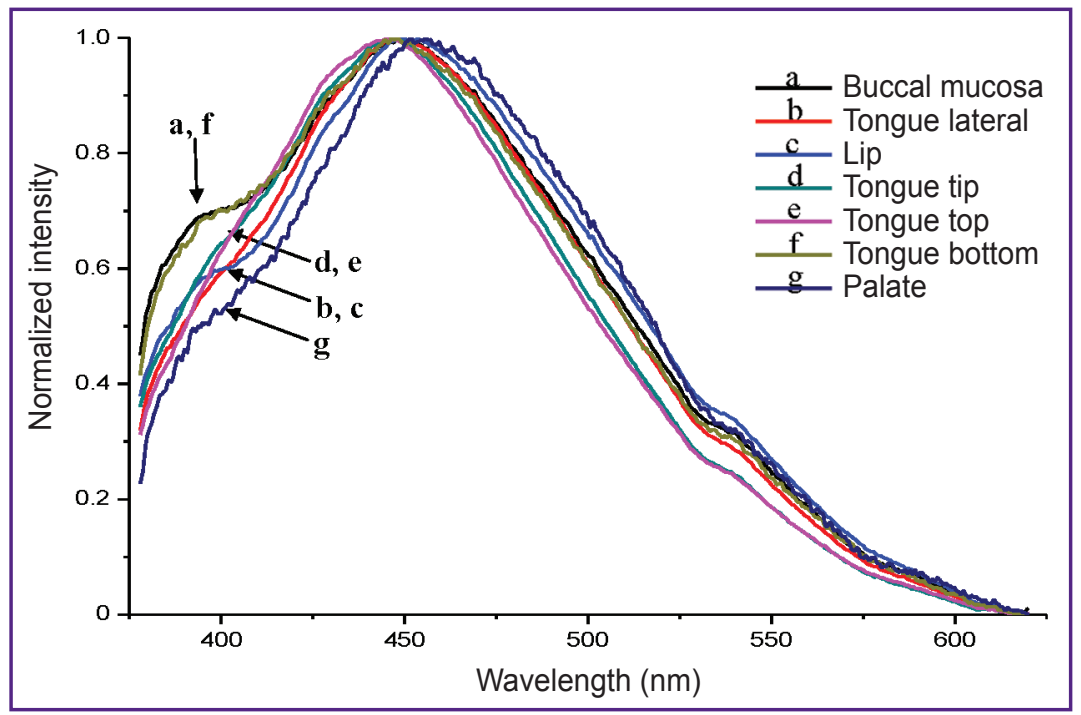

Figure 7. Mean spectra of different sites in malignant condition
Because of resultant broad distribution in the decision making parameters - in this case score of factor 1 through combining samples which may differ slightly, the distribution of the parameter will be broadened, making it difficult to decide the cut off/threshold value, necessary to include a test sample in a given class, in this case normal tissue. We will see later that similar considerations will apply for potentially malignant and malignant conditions also. In view of this, though lip (underside) and tongue bottom can be considered to form a combined reference data base with buccal mucosa, all the other sites (tongue tip, tongue top, tongue lateral, and palate) have to be treated separate, not only from buccal mucosa, but also from each other for more dependable classification.

Oral cavity sites - malignant conditions. Figure 7 shows the mean fluorescence spectra of different oral cavity sites under clinically malignant conditions. As before, if we look at the difference spectra (mean malignant buccal mucosa site - mean malignant other sites) we can get a better idea of the implications in clubbing together spectra from different sites. It is seen (Figure 8) that though buccal mucosa, tongue bottom, and lip underside gave very similar spectra in normal conditions, all sites, including lip underside (except tongue bottom), show noticeable differences from buccal mucosa, in malignancy. In view of this even for lip underside it is necessary to have separate reference data sets of normal and malignant conditions for diagnosis. Similarly, tongue tip, tongue lateral, tongue top, and palate also differ from each other in the 400-600 $\mathrm{nm}$ region, indicating that individual reference data sets are necessary for these sites for optical diagnostic applications.

Oral cavity sites - potentially malignant conditions. From the spectra of different oral cavity sites in clinically normal and malignant conditions, we have seen that there are 5-6 different molecular species contributing to the fluorescence spectra in the 375 $600 \mathrm{~nm}$ region. Also, except, may be for buccal mucosa and tongue bottom, it is advisable to have different sets of reference data bases for each site. These conclusions are further strengthened by study of potentially malignant conditions on the different sites. 
Figure 8. Difference spectra of malignant sites (mean malignant buccal mucosa mean malignant other site)

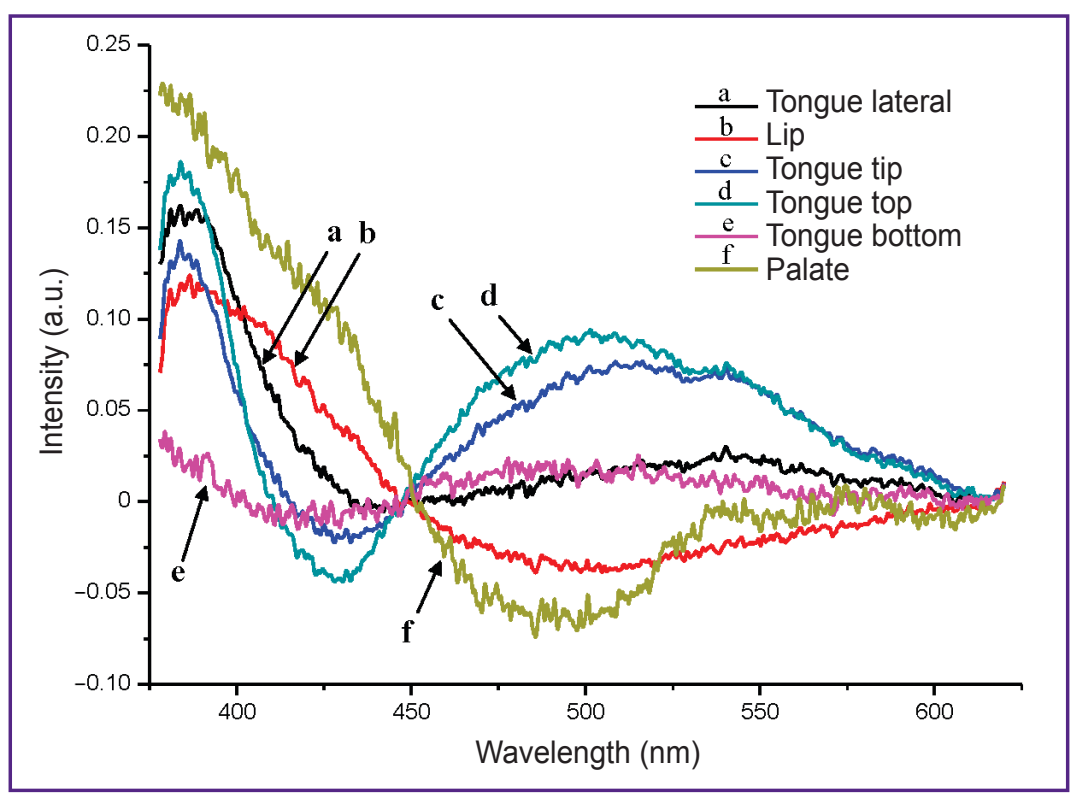

Oral malignancies are often preceded by potentially malignant conditions like leukoplakia, erythroplakia, and oral submucous fibrosis (OSMF). It is reported that about $25 \%$ of leukoplakia cases go over into squamous cell carcinoma [32]. As seen from Table 1 we had a reasonably large number of subjects for leukoplakia of buccal mucosa and lip, and for all sites (except palate) for OSMF. Figure 9 and Figure 10 show the mean spectra from different sites for leukoplakia and OSMF, respectively. Once again, it is seen that under same potentially malignant conditions different oral cavity sites give noticeably different spectra, which span a range of values in several regions. Clubbing together some (or all) of these together, will widen the distribution of diagnostic parameters, leading to larger uncertainties in the statistical reliability of diagnostic evaluation.

Fluorescent species in oral cavity. Müller et al. [11] have attributed spectra obtained by $337 \mathrm{~nm}$ excitation to only two species, collagen and $\mathrm{NADH}$, and fitted the observed fluorescence with fluorescence of these two species, modified with hemoglobin absorption. However, the presence of three clearly separate fluorescence peaks observed in tongue bottom, lower lip, tongue lateral, and buccal mucosa indicate that at least three different fluorescent species are present in these tissues. It is well known that bound $\mathrm{NADH}$, gives a spectrum at shorter wavelengths compared to free $\mathrm{NADH}$ and this may account for the peak around $430 \mathrm{~nm}$ [33]. Fluorescence spectra of endometrial curettage and other tissue specimens have also been analyzed by curve fitting with combinations of several bio-molecular species [34, 35].
Excitation in the $320-340 \mathrm{~nm}$ range produces an extended fluorescence spectrum spanning 350-650 nm (or more) range from oral cavity sites. The fluorescence has been attributed to superposition of fluorescence bands from several species, each giving rise to fairly broad (several tens of nanometers) fluorescence peaks. Under these conditions curve fitting techniques can give good fit of the observed spectrum, with a suitable combination of even two individual bands. It is therefore not very correct to use simple curve fitting methods for assignment of the observed spectrum to different fluorescent species. More important, using the bands of the pure components for fitting the actual spectrum can be only a poor approximation, since intermolecular interactions and environmental factors in the tissue can 


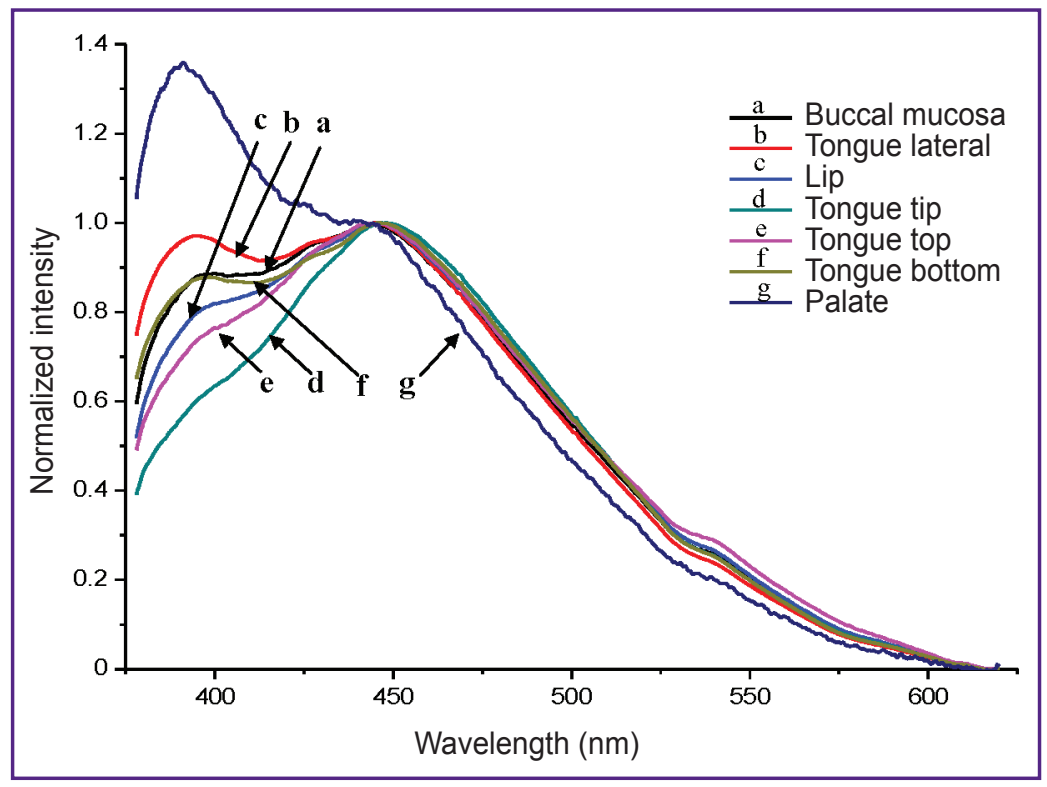

Figure 10. Mean spectra of oral sites in oral submucous fibrosis

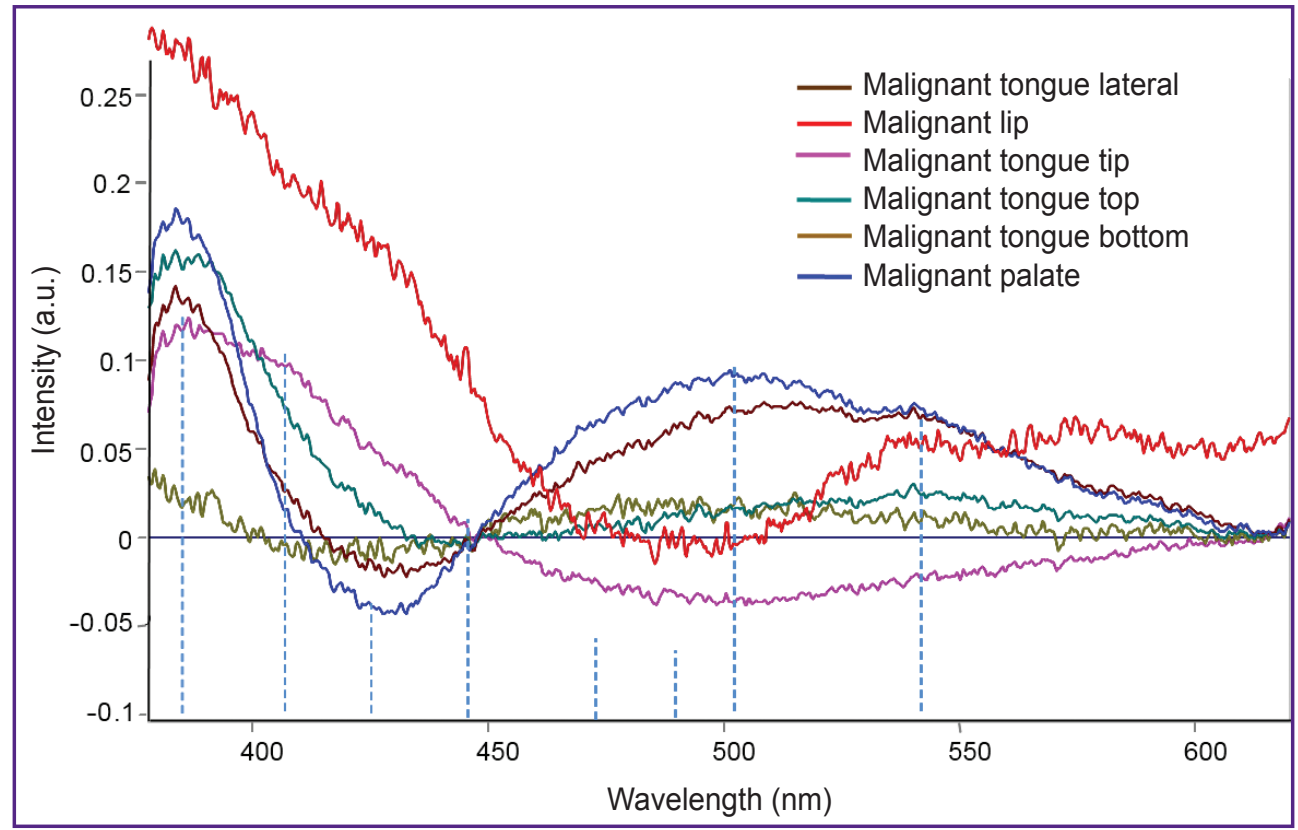

Figure 11. Difference spectra of leukoplakia condition (spectrum of buccal mucosa spectrum from other sites)

considerably alter the spectra of the pure components in the tissue. It is therefore necessary to have a good idea of the number of possible components before attempting any curve fit.

An idea of the number of components can be had from the difference spectra. As we have discussed earlier, in a difference spectrum, any change in concentration of a given species can give a difference band, positive or negative, depending on whether there is an increase or decrease in concentration of that species. Also, whenever two or more bands overlap in such a way that the overlapping produces a spectrum which looks like a single band for one site, in a difference spectrum they will get resolved into the individual components, because it is highly unlikely that concentrations of the two species producing the single-looking overlapping band, change in such a way that the appearance of the single looking band is unchanged for both sites.

As was seen from our difference spectra in Figures 4 and 8 these spectra do give a better idea of the number of independent bands. This is more clearly seen in Figure 11 which shows the difference spectra (spectrum of buccal mucosa - spectrum from other sites) for leukoplakia of the different sites. It is seen that there are at least 7-8 fluorescence bands in the spectra. It is also seen that the $500-600 \mathrm{~nm}$ region, in some cases gives a single, broad 
difference band while in other cases it shows two bands, one positive, and the other negative (see Figure 4) clearly showing that there are at least 3 bands in this region.

A careful examination of all our difference spectra, for different tissue conditions of the different oral cavity sites, gives an idea of the possible bands. It is to be noted that some of these peaks show slight changes under different conditions. Even if we assume these changes are due to different conditions of the tissue we still have at least 8 separate molecular species giving rise to the fluorescence. Table 2 gives the peak positions and probable assignments for these bands. Curve fitting of the spectra with the values of peak positions and half widths shown in Figure 12 gave very good fit for all sites.

Normal, potentially malignant, and malignant conditions of oral cavity sites screening and diagnosis. We have seen that the different sites of the oral cavity give noticeably different fluorescence spectra on excitation with $325 \mathrm{~nm}$, not only in the normal but also under potentially malignant and malignant conditions, making the use of a common reference data set not very advisable. Since the clinician knows which site he is examining, more important for diagnostic purposes are possible differences in different clinical conditions for the same site. As a typical example of such differences, in Figure 13 we show the mean spectra of buccal mucosa under different disease conditions, as difference spectra from normal buccal mucosa.

From Figure 13 it is clear that the different disease conditions of buccal mucosa give sufficiently different fluorescence spectra, which
Table 2

Peak positions and assignments of laser-induced fluorescence spectrum of buccal mucosa (Figure 12)

\begin{tabular}{ccccl}
\hline $\begin{array}{c}\text { Peak } \\
\text { No. }\end{array}$ & $\begin{array}{c}\text { Peak max. } \\
(\mathrm{nm})\end{array}$ & $\begin{array}{c}\text { Peak max. } \\
\left(\mathrm{cm}^{-1}\right)\end{array}$ & Area & \multicolumn{1}{c}{ Assignment } \\
\hline 1 & 391.36 & $25,552.04$ & 2012.91 & Collagen \\
\hline 2 & 425.14 & $23,521.70$ & 101.19 & Pyridoxine \\
\hline 3 & 445.50 & $22,446.70$ & 36.56 & Elastin \\
\hline 4 & 453.65 & $22,043.32$ & 3421.28 & Nicotinamide adenine dinucleotide \\
\hline 5 & 510.98 & $19,570.33$ & 485.82 & $\begin{array}{l}\text { Carotene (given wavelength }- \\
525 \text { nm) [34, 35] }\end{array}$ \\
\hline 6 & 552.42 & $18,102.06$ & 118.53 & Flavin adenine dinucleotide \\
\hline 7 & 586.65 & $17,045.99$ & 29.35 & New \\
\hline
\end{tabular}

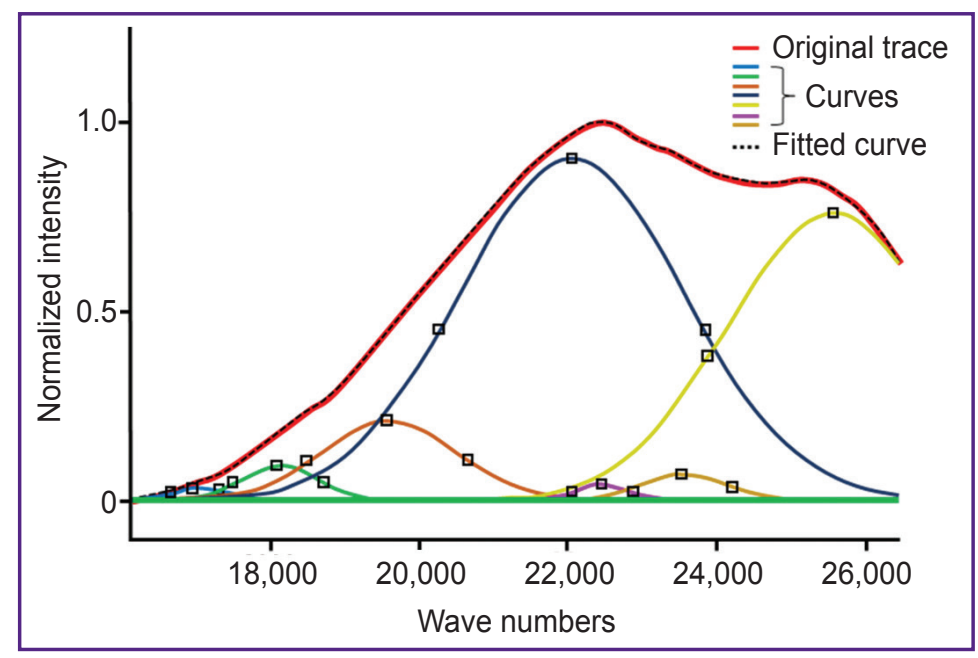

Figure 12. Curve fitting of laser-induced fluorescence spectrum of buccal mucosa showing the peak positions and assignments. The curves in seven colors stand for seven fluorophores
Figure 13. Difference spectra of normal buccal mucosa (normal buccal mucosa - buccal mucosa with different clinical conditions)

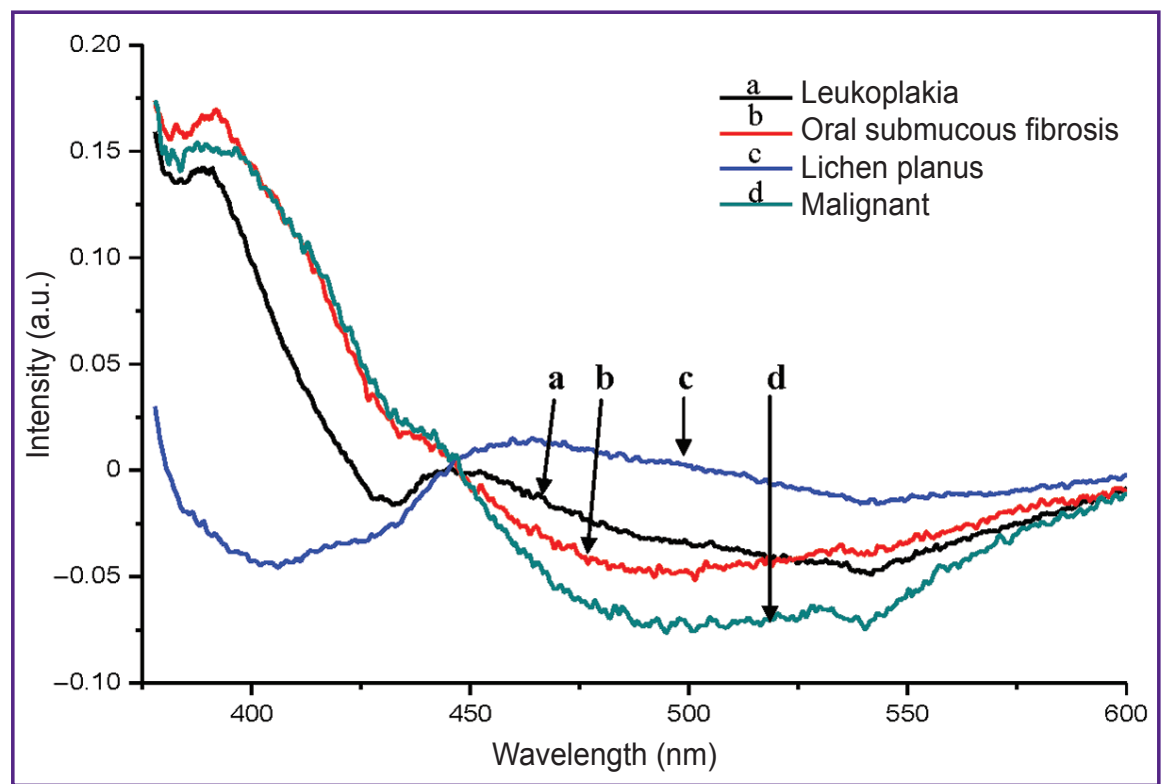




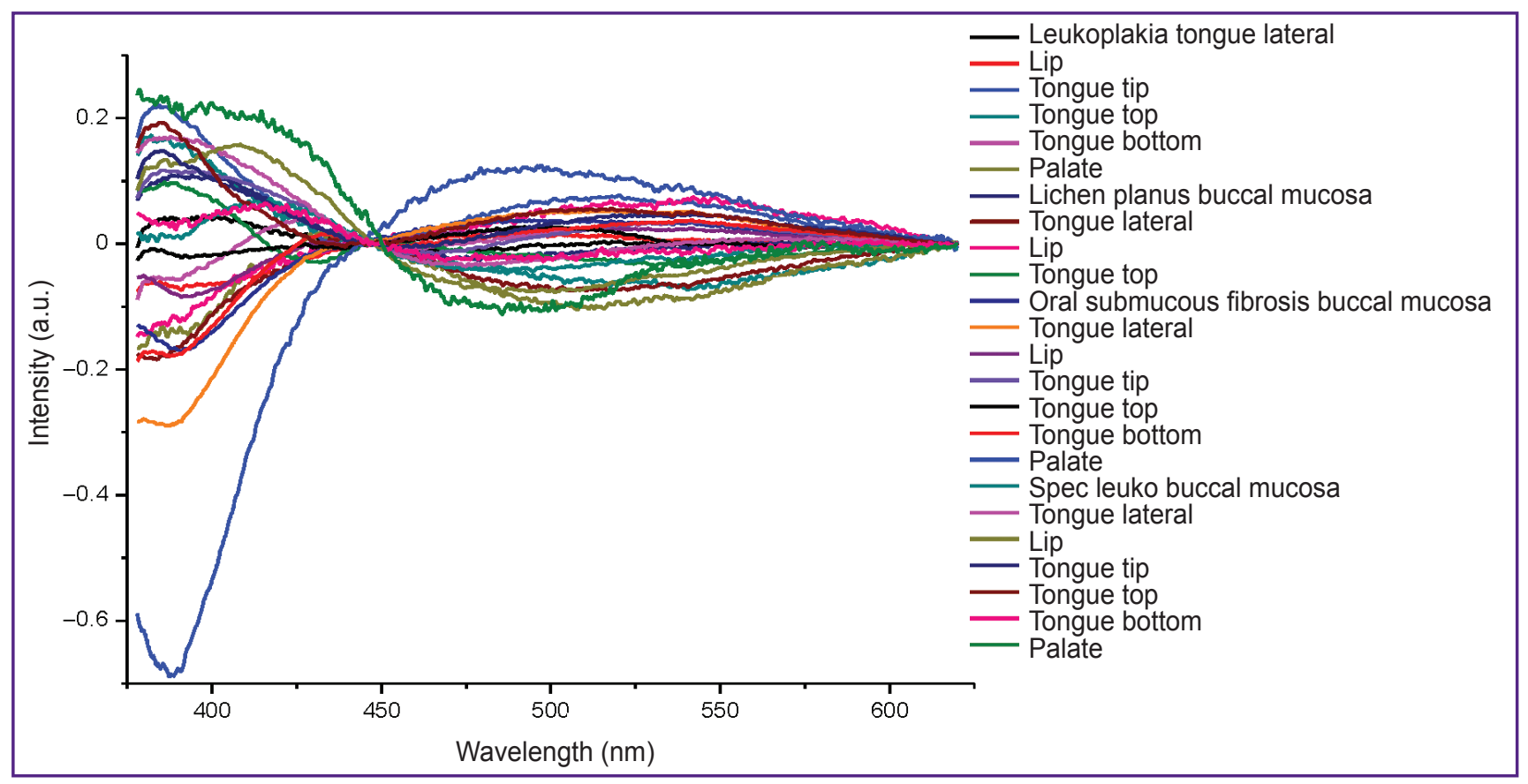

Figure 14. Scores of factor 1 for normal sites from the malignant and potentially malignant conditions

enable correct identification of the clinical condition making them quite suitable for diagnostic purposes. Similar results (Figure 14) were obtained for the other sites also.

It is seen from Table 1 that, except for buccal mucosa, only fewer subjects are available for potentially malignant and malignant conditions of the other sites. In view of this it is helpful to check whether combined reference data sets can be used for diagnostic applications. It was observed that the spectral changes at different sites, for different clinical conditions, vary over a wide range and combining them to form a single reference data set is not advisable because of the possible wide dispersion in decision making parameters with such a combined set.

Optical biopsy: screening in oral malignancy. The identification of potentially malignant/malignant conditions is usually done at present by biopsy and histopathology. But the effects of field cancerization leading to multicentric lesions, inter-observer and intra-observer variations in reading the degree of epithelial dysplasia, and the possibility that a biopsy may not be representative of the whole lesion, lead to the high likelihood of a biopsy not being reliable for diagnosis [36]. It is thus essential that potentially malignant conditions in oral cavity should be followed by regular periodic observations. Obviously, biopsy is not suitable for this purpose. Optical biopsy by spectroscopic methods, which are non-invasive, highly objective, and operator-independent is ideal for this purpose. The usefulness of the fluorescence optical technique can be appreciated by a look at the spectra of oral cavity sites of susceptible population, mainly tobacco users. The fact that tissue alterations have already been taking place in such cases is observed by changes in fluorescence spectra of tissue samples, diagnosed as normal by histopathology, from subjects under malignant and potentially malignant conditions. To confirm this, we determined the scores for the mean spectra of samples judged to be normal visually by the clinician, for all the sites, with various clinical conditions, adding them as test samples to the normal buccal mucosa set. The results are shown in Figure 14. It is seen that tissue sections adjacent to lesion sites and considered to be normal by clinician, give factor 1 values closely similar to malignant spectra for buccal mucosa, lip, tongue bottom and the other sites. If such conditions are observed during routine screening of susceptible groups (tobacco users) through LIF, remedial action can be taken (advise the patients to quit tobacco smoking, administer the required therapy) early enough, without any need for biopsy and histopathology. Optical biopsy by LIF is quite suitable for this purpose. Though clinical samples like tissue contain many biomolecules only a few are present in relatively large amounts. Under normal conditions they will all be present within specific concentration ranges in all samples of a given type, for example, buccal mucosa. Further, at the shorter wavelengths used for fluorescence spectroscopy, the radiation penetrates only a few hundred microns, and the resultant fluorescence, when measured at the illumination point, will be mostly from this top layer [31]. It is thus possible to represent the total profile as a sum of fluorescence profiles of combinations of 2-3 species, each combination varying only slightly from sample to sample. The consequence of this is that if we take the mean of several profiles of a given class, buccal mucosa from normal subjects, each individual member of the class will differ from the mean only by small amounts. 


\section{Conclusions}

In healthy conditions (from subjects with no abnormal oral conditions, including tobacco use), buccal mucosa, lip underside, and tongue bottom give spectra very similar to each other, while tongue top, tongue lateral, and palate give spectra differing from these as well as from each other. The curve fitting analysis has shown that the variations in the spectra may be due to the presence of 7-8 fluorophores with varying concentrations. Under potentially malignant and malignant conditions all sites give spectra which are noticeably different for different sites in different conditions. It is the usual practice that common spectra are being used for comparison of normal oral tissue with respect to disease conditions of oral cavity. It is therefore not advisable to use common reference data sets of normal, potentially malignant, or malignant conditions of different sites for diagnostic applications. By matching test samples to standard calibration sets for each anatomical site, for different conditions, accurate diagnosis can be achieved for potentially malignant and malignant states, with high sensitivity and specificity. The fluorescence spectroscopy technique can thus be routinely employed for regular screening of susceptible population groups, for diagnostic applications to discriminate potentially malignant and malignant conditions, and for follow up in therapy, without the need for repeated biopsy.

Acknowledgement. Authors are thankful to Manipal Academy of Higher Education, Manipal, India for providing the research facility for the work and the Department of Science and Technology, Government of India for the project titled "Design, Assembly, Testing and Multi-Centre Evaluation of a Miniature and Portable LIF System for Screening of Oral Malignancy, Ref. No.IDP/MED/2013/03 dated 02/06/2015".

Conflict of Interests. The authors have no conflict of interests to disclose.

\section{References}

1. Alfano R., Tata D., Cordero J., Tomashefsky P., Longo F., Alfano M. Laser induced fluorescence spectroscopy from native cancerous and normal tissue. IEEE J Quantum Electron 1984; 20(12): 1507-1511, https://doi.org/10.1109/ jqe.1984.1072322.

2. Wagnieres G.A., Star W.M., Wilson B.C. In vivo fluorescence spectroscopy and imaging for oncological applications. Photochem Photobiol 1998; 68(5): 603-632, https://doi.org/10.1111/j.1751-1097.1998.tb02521.x.

3. Gillenwater A., Jacob R., Ganeshappa R., Kemp B., El-Naggar A.K., Palmer J.L., Clayman G., Mitchell M.F., Richards-Kortum R. Noninvasive diagnosis of oral neoplasia based on fluorescence spectroscopy and native tissue autofluorescence. Arch Otolaryngol Head Neck Surg 1998; 124(11): 1251-1258, https://doi.org/10.1001/ archotol.124.11.1251.

4. Chen C.T., Chiang H.K., Chow S.N., Wang C.Y., Lee Y.S., Tsai J.C., Chiang C.P. Autofluorescence in normal and malignant human oral tissues and in DMBA-induced hamster buccal pouch carcinogenesis. J Oral Pathol Med 1998; 27(10): 470-474, https://doi.org/10.1111/j.1600-0714.1998.tb01914.x.

5. Inaguma M., Hashimoto K. Porphyrin-like fluorescence in oral cancer. Cancer 1999; 86(11): 2201-2211, https://doi. org/10.1002/(sici)1097-0142(19991201)86:11<2201::aidcncr5>3.0.co;2-9.

6. Jayanthi J.L., Subhash N., Stephen M., Philip E.K., Beena V.T. Comparative evaluation of the diagnostic performance of autofluorescence and diffuse reflectance in oral cancer detection: a clinical study. J Biophotonics 2011; 4(10): 696-706, https://doi.org/10.1002/jbio.201100037.

7. Wang C.Y., Chen C.T., Chiang C.P., Young S.T., Chow S.N., Chiang H.K. A probability-based multivariate statistical algorithm for autofluorescence spectroscopic identification of oral carcinogenesis. Photochem Photobiol 1999; 69(4): 471-477, https://doi.org/10.1111/j.1751-1097.1999. tb03314.x.

8. Heintzelman D.L., Utzinger U., Fuchs H., Zuluaga A., Gossage K., Gillenwater A.M., Jacob R., Kemp B., RichardsKortum R.R. Optimal excitation wavelengths for in vivo detection of oral neoplasia using fluorescence spectroscopy. Photochem Photobiol 2000; 72(1): 103-113, https://doi. org/10.1562/0031-8655(2000)072<0103:oewfiv>2.0.co;2.

9. Majumder S.K., Mohanty S.K., Ghosh N., Gupta P.K., Jain D.K., Khan F. A pilot study on the use of autofluorescence spectroscopy for diagnosis of the cancer of human oral cavity. Current Science 2000; 79(8): 1089-1094.

10. Eker C., Rydell R., Svanberg K., Andersson-Engels S. Multivariate analysis of laryngeal fluorescence spectra recorded in vivo. Lasers Surg Med 2001; 28(3): 259-266, https://doi.org/10.1002/lsm.1048.

11. Müller M.G., Valdez T.A., Georgakoudi I., Backman V., Fuentes C., Kabani S., Laver N., Wang Z., Boone C.W., Dasari R.R., Shapshay S.M., Feld M.S. Spectroscopic detection and evaluation of morphologic and biochemical changes in early human oral carcinoma. Cancer 2003; 97(7): 1681-1692, https://doi.org/10.1002/cncr.11255.

12. Ebihara A., Krasieva T.B., Liaw L.H., Fago S., Messadi D., Osann K., Wilder-Smith P. Detection and diagnosis of oral cancer by light-induced fluorescence. Lasers Surg Med 2003; 32(1): 17-24, https://doi.org/10.1002/lsm.10137.

13. Wang C.-Y., Tsai T., Chen H.-M., Chen C.-T., Chiang C.-P. PLS-ANN based classification model for oral submucous fibrosis and oral carcinogenesis. Lasers Surg Med 2003; 32(4): 318-326, https://doi.org/10.1002/lsm.10153.

14. De Veld D.C.G., Skurichina M., Witjes M.J.H., Duin R.P.W., Sterenborg D.J.C.M., Star W.M., Roodenburg J.L. Autofluorescence characteristics of healthy oral mucosa at different anatomical sites. Lasers Surg Med 2003; 32(5): 367376, https://doi.org/10.1002/lsm.10185.

15. Tsai T., Chen H.-M., Wang C.-Y., Tsai J.-C., Chen C.-T., Chiang C.-P. In vivo autofluorescence spectroscopy of oral premalignant and malignant lesions: distortion of fluorescence intensity by submucous fibrosis. Lasers Surg Med 2003; 33(1): 40-47, https://doi.org/10.1002/lsm.10180.

16. Majumder S.K., Ghosh N., Kataria S., Gupta P.K. Nonlinear pattern recognition for laser-induced fluorescence diagnosis of cancer. Lasers Surg Med 2003; 33(1): 48-56, https://doi.org/10.1002/lsm.10191.

17. Manjunath B.K., Kurein J., Rao L., Krishna C.M., Chidananda M.S., Venkatakrishna K., Kartha V.B. Autofluorescence of oral tissue for optical pathology in oral 
malignancy. J Photochem Photobiol B 2004; 73(1-2): 49-58, https://doi.org/10.1016/j.jphotobiol.2003.09.004.

18. Skandarajah A., Sunny S.P., Gurpur P., Reber C.D., D'Ambrosio M.V., Raghavan N., James B.L., Ramanjinappa R.D., Suresh A., Kandasarma U., Birur P., Kumar V.V., Galmeanu H.C., Itu A.M., Modiga-Arsu M., Rausch S., Sramek M., Kollegal M., Paladini G., Kuriakose M., Ladic L., Koch F., Fletcher D. Mobile microscopy as a screening tool for oral cancer in India: a pilot study. PLoS One 2017; 12(11): e0188440, https://doi.org/10.1371/journal.pone.0188440.

19. De Veld D.C., Sterenborg H.J.C., Roodenburg J.L., Witjes M.J. Effects of individual characteristics on healthy oral mucosa autofluorescence spectra. Oral Oncology 2004; 40(8): 815-823, https://doi.org/10.1016/j.oraloncology.2004.02.006.

20. Mallia R., Thomas S.S., Mathews A., Kumar R., Sebastian P., Madhavan J., Subhash N. Oxygenated hemoglobin diffuse reflectance ratio for in vivo detection of oral pre-cancer. J Biomed Opt 2008; 13(4): 041306, https://doi. org/10.1117/1.2952007.

21. di Fiore M.S.H. Atlas of normal histology. Edited by V.P. Eroschenko. K.M. Varghese Co; Bombay, 1990.

22. Unnikrishnan V.K., Nayak R., Bernard R. Priya K.J., Patil A., Ebenezer J., Pai K.M., George S.D., Kartha V.B., Santhosh C. Parameter optimization of a laser-induced fluorescence system for in vivo screening of oral cancer. J Laser Appl 2011; 23(3): 032004, https://doi. org/10.2351/1.3591342.

23. Patil A., Rao K.S., Unnikrishnan V.K., Pai K.M., Kartha V.B., Chidangil S. Development of a miniature autofluorescence device for the early diagnosis of squamous cell carcinoma. Proc. SPIE 10411, Clinical and Preclinical Optical Diagnostics 2017, 104110V, https://doi. org/10.1117/12.2282888.

24. Krishna C.M., Sockalingum G.D., Kurien J., Rao L., Venteo L., Pluot M., Manfait M., Kartha V.B. Micro-Raman spectroscopy for optical pathology of oral squamous cell carcinoma. Appl Spectrosc 2004; 58(9): 1128-1135.

25. Malini R., Venkatakrishna K., Kurien J., Pai K.M., Rao L., Kartha V.B., Krishna C.M. Discrimination of normal, inflammatory, premalignant, and malignant oral tissue: a Raman spectroscopy study. Biopolymers 2006; 81(3): 179193, https://doi.org/10.1002/bip.20398.

26. Kartha V.B., Kurien J., Rai L., Mahato K.K., Krishna C.M.,
Santhosh C. Diagnosis at the molecular level: analytical laser spectroscopy for clinical applications. In: Kaneco S., Viswanathan B., Funasaka K. Photoelectrochemistry and photobiology in environment, energy and fuel. Research Signapost, Trivendrum; India, 2005; p. 153-221.

27. Patil A., Prabhu V., Choudhari K.S., Unnikrishnan V.K., George S.D., Ongole R., Pai K.M., Shetty J.K., Bhat S., Kartha V.B., Chidangil S. Evaluation of high-performance liquid chromatography laser-induced fluorescence for serum protein profiling for early diagnosis of oral cancer. J Biomed Opt 2010; 15(6): 067007, https://doi.org/10.1117/1.3523372.

28. Bhat S., Patil A., Rai L., Kartha V.B., Santhosh C. Protein profile analysis of cellular samples from the cervix for the objective diagnosis of cervical cancer using HPLC-LIF. J Chromatogr B Analyt Technol Biomed Life Sci 2010; 878(31): 3225-3230, https://doi.org/10.1016/j.jchromb.2010.09.025.

29. Bhat S., Patil A., Rai L., Kartha V.B., Chidangil S. Application of HPLC combined with laser induced fluorescence for protein profile analysis of tissue homogenates in cervical cancer. The Scientific World Journal 2012; 2012: 976421 https://doi.org/10.1100/2012/976421

30. Venkatakrishna K., Kartha V.B., Pai K.M., Krishna C.M., Ravikiran O., Kurian J., Alexander M., Ullas G. HPLC-LIF for early detection of oral cancer. Current Science 2003; 84(4): 551-557.

31. Patil A., Choudhari K.S., Unnikrishnan V.K., Shenoy N., Ongole R., Pai K.M., Kartha V.B., Chidangil S. Salivary protein markers: a noninvasive protein profile-based method for the early diagnosis of oral premalignancy and malignancy. J Biomed Opt 2013; 18(10): 101317, https://doi.org/10.1117/1. jbo.18.10.101317.

32. Chance B. Photon migration in tissues. Springer; 1990.

33. Lackowicz J.R. Principles of fluorescence spectroscopy. Vol. 1. Springer, 2006.

34. Gavryushin V., Vaitkus J., Vaitkuviene A. Methodology of medical diagnostics of human tissue by fluorescence. Lithuania J Physics 2000; 40(4): 232-236.

35. Gavryushin V., Vaitkuvienè A. Modelling studies of alive tissue fluorophores. Lithuania J Physics 2003.

36. Holmstrup P., Vedtofte P., Reibel J., Stoltze K. Oral premalignant lesions: is a biopsy reliable? J Oral Pathol Med 2007; 36(5): 262-266, https://doi.org/10.1111/j.16000714.2007.00513.x. 